\title{
AUTOMORPHISM GROUPS OF DIFFERENTIABLE DOUBLE LOOPS
}

Dedicated to Karl H. Hofmann, on the occasion of his 60 th birthday

\begin{abstract}
ARSTRACT. In this paper, we study local and global topological loops as well as topological double loops having a differentiable structure such that the loop operations are differentiable. The main result states that the group of differentiable automorphisms of a differentiable double loop is compact with respect to the compact-open topology.
\end{abstract}

The automorphism group $\Gamma$ of a locally compact connected double loop $\mathscr{D}$ is a locally compact topological group, where the group $\Gamma$ will always be provided with the compact-open topology. For a proof of this result see [1]. If $\mathscr{D}$ is even a Cartesian field, in particular if $\mathscr{D}$ is one of the classical double loops $\mathbb{R}, \mathbb{C}, \mathbb{H}$, or $\mathbb{D}$, then $\Gamma$ is a compact Lie group. In general, it is an open problem whether the group $\Gamma$ is compact or a Lie group. But the four classical double loops are not merely topological double loops; they also possess a differentiable structure such that the loop operations are differentiable. In a recent paper, J. Kozma has shown that the automorphism group of a $\mathbf{C}^{2}$-loop can be embedded into a linear group (see [7] or Theorem (2.1) below). The aim of this paper is to give an appropriate definition of a differentiable double loop and to prove that the automorphism group of such a double loop can be embedded as a closed subgroup into a linear group. Using this embedding theorem, we are able to verify the compactness of the automorphism group.

\section{Definitions AND NOTATION}

(1.1) DEFINITION. A quadruple $\mathscr{L}=(L, U, 0,+)$ is called a local $H$-space if the following conditions are satisfied:

(1) $L$ is a topological space.

(2) $U$ is an open neighborhood of the element $0 \in L$.

(3) There exists an open neighborhood $V$ of 0 in $U$ such that the map $+: V \times V \rightarrow U$ is continuous.

(4) $x+0=0+x=x$ for every $x \in V$.

The neighborhood $V$ is called the support of $\mathscr{L}$. 
A local $H$-space $\mathscr{L}=(L, U, 0,+)$ with support $V$ is called a local loop iff the following statements hold:

(5a) For all $a, x, y \in V$ the equation $a+x=a+y \in U$ implies that $x=y$.

(5b) For all $a, x, y \in V$ the equation $x+a=y+a \in U$ implies that $x=y$.

Note that in the definition of a local loop we do not require that the local inverses of the operation + are continuous.

(1.2) DEFINITION. A local $H$-space $\mathscr{L}=(L, U, 0,+)$ is called a smooth local $H$-space of dimension $n$ iff

(1) $U$ is an $n$-dimensional $\mathbf{C}^{2}$-manifold,

(2) there is an open neighborhood $V \subseteq U$ of 0 such that $+: V \times V \rightarrow U$ is a $\mathrm{C}^{2}$-mapping.

The neighborhood $V$ is again called the support of $\mathscr{L}$.

A local loop $\mathscr{L}=(L, U, 0,+)$ is called a smooth local loop iff $\mathscr{L}$ is a smooth local $H$-space.

Note that if $\mathscr{L}=(L, U, 0,+)$ is a smooth local loop, then $\mathscr{L}=\left(L, U^{\prime}, 0,+\right)$ is also a smooth local loop for any neighborhood $U^{\prime} \subseteq U$ of the element 0 .

The following result of J. P. Holmes and A. A. Sagle ([4, Th. 1.1]) shows that in the differentiable case the notion of a local $H$-space coincides with the notion of a local loop.

(1.3) THEOREM. A smooth local $H$-space is always a (smooth) local loop.

(1.4) DEFINITION. Let $\mathscr{L}=(L, U, 0,+)$ be a smooth local loop of dimension $n$ with support $V$. A $\mathbf{C}^{2}$-diffeomorphism $h: U \rightarrow \mathbb{R}^{n}$ satisfying $h(0)=0$ is called a smooth coordinate system of $\mathscr{L}$. A smooth coordinate system $h$ of $\mathscr{L}$ is called canonical iff there is a star-shaped neighborhood $S \subseteq h(V)$ of 0 such that for every $x \in S$ the relation

$$
h^{-1}(x)+h^{-1}(x)=h^{-1}(2 x)
$$

is satisfied.

The main tool for our investigations is a recent result by J. Kozma, which we state explicitly.

(1.5) THEOREM. Every smooth local loop has a smooth canonical coordinate system.

For a proof see [6, Th. 1]. 
(1.6) DEFINITION. Let $\mathscr{L}=(L, U, 0,+)$ be a smooth local loop of dimension $n$ with support $V$. Let $h$ be a smooth canonical coordinate system of $\mathscr{L}$ according to Theorem (1.5). Set $\mathscr{U}:=h(U)$ and $\mathscr{V}:=h(V)$. Then

$$
\oplus: \mathscr{V} \times \mathscr{V} \rightarrow \mathscr{U}:(h(x), h(y)) \mapsto h(x+y)
$$

defines a $\mathbf{C}^{2}$-mapping $\oplus$, and $\left(\mathbb{R}^{n}, \mathscr{U}, 0, \oplus\right)$ becomes a smooth local loop of dimension $n$ with support $\mathscr{V}$. This loop is called the induced loop of the canonical coordinate system $h$.

(1.7) DEFINITION. Let $\mathscr{L}=(L, U, 0,+)$ and $\mathscr{L}^{\prime}=\left(L^{\prime}, U^{\prime}, 0^{\prime},+^{\prime}\right)$ be smooth local loops with supports $V$ and $V^{\prime}$ respectively. A map $\gamma: W \rightarrow V^{\prime}$ which is defined on a neighborhood $W \subseteq V$ of 0 is called a homomorphism iff it is a $\mathbf{C}^{1}$ mapping (cf. the remark after (2.1)) satisfying $\gamma(x+y)=\gamma(x)+{ }^{\prime} \gamma(y)$ for all $x, y \in W$.

If $\mathscr{L}=\mathscr{L}^{\prime}$ and if $\gamma$ is an injective homomorphism of $\mathscr{L}$ such that the inverse mapping $\gamma^{-1}$ is also a homomorphism of $\mathscr{L}$, then the map $\gamma$ is called an automorphism of $\mathscr{L}$. Two automorphisms $\gamma, \gamma^{\prime}$ of $\mathscr{L}$ are called equivalent if and only if there is an open neighborhood $W$ of 0 such that $\gamma$ and $\gamma^{\prime}$ coincide on $W$.

(1.8) NOTATION. Let $\mathscr{L}=(L, U, 0,+)$ be a smooth local loop. The set of all automorphisms of $\mathscr{L}$ is denoted by $\operatorname{Aut}_{\text {loc }}^{*}(\mathscr{L})$. For $\gamma \in \operatorname{Aut}_{\text {loc }}^{*}(\mathscr{L})$ the set of all

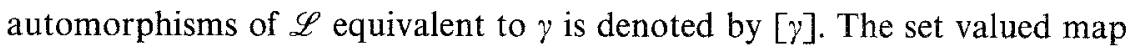
[] defines an equivalence relation on $\operatorname{Aut}_{\text {loc }}^{*}(\mathscr{L})$. Setting $\operatorname{Aut}_{\text {loc }}(\mathscr{L}):=$ Aut ${ }_{\text {loc }}^{*}(\mathscr{L}) /[]$, it is easily verified that $\left[\gamma_{1}\right]\left[\gamma_{2}\right]:=\left[\gamma_{1} \gamma_{2}\right]$ defines a group operation on $\mathrm{Aut}_{\mathrm{loc}}(\mathscr{L})$.

(1.9) DEFINITION. Let $\mathscr{L}=(L, 0,+)$ be a (global) topological loop in the ordinary sense, see [3], e.g. If there is an open neighborhood $U \subseteq L$ of the neutral element 0 such that $\mathscr{L}_{U}=(L, U, 0,+)$ is a smooth local loop, the local loop $\mathscr{L}_{U}$ is called a localization of the (global) loop $\mathscr{L}$. The loop $\mathscr{L}$ itself is then called a smooth loop.

(1.10) DEFINITION. Let $\mathscr{L}=(L, 0,+)$ be a smooth loop. Let $\gamma$ be a (global) continuous automorphism of $\mathscr{L}$. The automorphism $\gamma$ is called locally smooth if there is a localization $\mathscr{L}_{U}$ such that the restriction of $\gamma$ to $U$ lies in the set $\mathrm{Aut}_{\mathrm{loc}}^{*}\left(\mathscr{L}_{U}\right)$. The set of all locally smooth automorphisms of $\mathscr{L}$ is denoted by $\operatorname{Aut}^{1}(\mathscr{L})$.

Clearly, this set is a subgroup of the group $\operatorname{Aut}(\mathscr{L})$ of all continuous automorphism of $\mathscr{L}$. The group $\operatorname{Aut}^{1}(\mathscr{L})$ becomes a topological transformation group on $\mathscr{L}$ when provided with the compact-open topology. This topology coincides with the relative topology induced by the compact-open 
topology of $\operatorname{Aut}(\mathscr{L})$. We shall always take the group $\operatorname{Aut}^{1}(\mathscr{L})$ with the compact-open topology.

\section{Results}

In our study of the automorphism group $\operatorname{Aut}^{1}(\mathscr{L})$ of a smooth loop $\mathscr{L}$, i.e. of a loop $\mathscr{L}$ having a smooth localization, we shall often use the following fact, which again was proved by J. Kozma, see [7, Th., p. 500].

(2.1) THEOREM. Let $\mathscr{L}=(L, U, 0,+)$ be a smooth local loop of dimension $n$. Let $h$ be a canonical coordinate system with induced loop $\left(\mathbb{R}^{n}, \mathscr{U}, 0, \oplus\right)$. Let $\gamma \in \mathrm{Aut}_{\mathrm{loc}}^{*}(\mathscr{L})$ and let $\mathscr{W} \subseteq \mathscr{U}$ denote the domain of the mapping

$$
\Phi_{\gamma}: \mathscr{W} \rightarrow \mathbb{R}^{n}: x \mapsto h y h^{-1}(x)
$$

Then the set $\mathscr{W}$ is an open neighborhood of 0 , and the relation $\Phi_{\gamma}=\left.\Phi_{\gamma}^{\prime}(0)\right|_{\mathscr{W}}$ holds.

REMARK. Theorem (2.1) shows that an automorphism in $\mathrm{Aut}_{\mathrm{loc}}^{*}(\mathscr{L})$ is in fact a local $\mathbf{C}^{2}$-mapping, and so it is justified to call the elements of $\operatorname{Aut}_{\text {loc }}^{*}(\mathscr{L})$ smooth. More general, if $\mathscr{L}$ is a $\mathrm{C}^{k}$-loop for $k \geqslant 2$, then an automorphism in $\operatorname{Aut}_{\mathrm{loc}}^{*}(\mathscr{L})$ is indeed a $\mathbf{C}^{k}$-mapping.

(2.2) COROLLARY. Let $\mathscr{L}=(L, U, 0,+)$ be a smooth local loop of dimension $n$ with canonical coordinate system $h$. Then the map

$$
\Phi: \operatorname{Aut}_{\mathrm{loc}}^{1}(\mathscr{L}) \rightarrow \mathrm{GL}_{n} \mathbb{R}:[\gamma] \mapsto \Phi_{\gamma}^{\prime}(0)
$$

is an embedding of groups.

Proof. The map $\Phi$ is well-defined by Theorem (2.1). Let $\left[\gamma_{1}\right]$, $\left[\gamma_{2}\right] \in \operatorname{Aut}_{\text {loc }}^{1}(\mathscr{L})$. If $\Phi\left(\left[\gamma_{1}\right]\right)=\Phi\left(\left[\gamma_{2}\right]\right)$, then by Theorem (2.1) there exists a neighborhood $\mathscr{O} \subseteq \mathbb{R}^{n}$ of 0 satisfying

$$
\left.h \gamma_{1} h^{-1}\right|_{\mathscr{O}}=\left.\Phi_{\gamma_{1}}{ }^{\prime}(0)\right|_{\mathscr{O}}=\left.\Phi_{\gamma_{2}}{ }^{\prime}(0)\right|_{\mathscr{O}}=\left.h \gamma_{2} h^{-1}\right|_{\mathscr{O}} \text {. }
$$

Setting $O:=h^{-1}(\mathcal{O})$, this implies that $h \gamma_{1}$ and $h \gamma_{2}$ coincide on $O$. Since $h$ is injective on $O \subseteq U$, we conclude that $\gamma_{1}$ and $\gamma_{2}$ coincide on $O$. This means that $\left[\gamma_{1}\right]=\left[\gamma_{2}\right]$, because $O=h^{-1}(\mathcal{O})$ is a neighborhood of 0 in $\mathscr{L}$. This proves the injectivity of $\Phi$. The map $\Phi$ is a group homomorphism because

$$
\begin{aligned}
\Phi\left(\left[\gamma_{1}\right]\left[\gamma_{2}\right]\right) & =\Phi\left(\left[\gamma_{1} \gamma_{2}\right]\right)=\left(h\left(\gamma_{1} \gamma_{2}\right) h^{-1}\right)^{\prime}(0)=\left(\left(h \gamma_{1} h^{-1}\right)\left(h \gamma_{2} h^{-1}\right)\right)^{\prime}(0) \\
& =\left(h \gamma_{1} h^{-1}\right)^{\prime}(0) \cdot\left(h \gamma_{2} h^{-1}\right)^{\prime}(0)=\Phi\left(\left[\gamma_{1}\right]\right) \cdot \Phi\left(\left[\gamma_{2}\right]\right) .
\end{aligned}
$$

Thus the mapping $\Phi$ is an embedding of groups.

Next we investigate the case of a connected loop $\mathscr{L}=(L, 0,+)$ which has a 
smooth localization $\mathscr{L}_{U}$ of dimension $n$. It turns out that the group $\operatorname{Aut}^{1}(\mathscr{L})$ can be embedded in $\mathrm{GL}_{n} \mathbb{R}$ even as a topological group.

(2.3) COROLLARY. Let $\mathscr{L}=(L, 0,+)$ be a connected loop which has a smooth localization $\mathscr{L}_{U}$ of dimension $n$. Then the map

$$
\Psi: \operatorname{Aut}^{1}(\mathscr{L}) \rightarrow \mathrm{GL}_{n} \mathbb{R}: \gamma \mapsto \Phi_{\gamma}^{\prime}(0)
$$

is an embedding of topological groups.

Proof. Let $h$ be a canonical coordinate system of $\mathscr{L}_{U}$ with induced loop $\left(\mathbb{R}^{n}, \mathscr{U}, 0, \oplus\right)$. We shall divide the proof into three steps. In step (1) we describe an appropriate neighborhood basis of the identity in $\mathrm{GL}_{n} \mathbb{R}$, which we need in steps (2) and (3), where the continuity and the openness of the mapping $\Psi$ is proved.

(1) The family of sets $\Omega(\mathscr{K}, \mathcal{O})=\left\{g \in \mathrm{GL}_{n} \mathbb{R} ; g(\mathscr{K}) \subseteq \mathcal{O}\right\}$ with $\mathscr{K} \subseteq \mathcal{O} \subseteq \mathscr{U}$, where $\mathscr{K}$ is compact and $\mathscr{O}$ is an open set, constitutes a neighborhood basis of the identity element $\mathbb{1} \in \mathrm{GL}_{n} \mathbb{R}$.

This follows immediately from the definition of the compact-open topology and linearity.

(2) The map $\Psi: \operatorname{Aut}^{1}(\mathscr{L}) \rightarrow \mathrm{GL}_{n} \mathbb{R}: \gamma \mapsto \Phi_{\gamma}{ }^{\prime}(0)=\left(h \gamma h^{-1}\right)^{\prime}(0)$ is continuous.

Since the loop $\mathscr{L}$ is connected, it is generated by any neighborhood $W$ of $L$, i.e. the smallest closed subloop of $\mathscr{L}$ containing $W$ is $\mathscr{L}$ itself, see [3, (3.1), (3.2)]. Thus, two automorphisms of $\mathscr{L}$ coincide, if they coincide on an arbitrary neighborhood (of 0 ) and hence $\Psi$ is a group monomorphism by Corollary (2.2). So it remains to verify the continuity of $\Psi$ at the identity. For that, select a neighborhood $\Omega(\mathscr{K}, \mathcal{O})$ of $\mathbb{1} \in \mathrm{GL}_{n} \mathbb{R}$. By step (1) we may assume that $\mathscr{K} \subseteq \mathcal{O} \subseteq \mathscr{U}$. To check the continuity of $\Psi$ at the identity map, we shall verify the inclusion

$$
\Psi\left(\Omega\left(h^{-1}(\mathscr{K}), h^{-1}(\mathcal{O})\right)\right) \subseteq \Omega(\mathscr{K}, \mathcal{O}) .
$$

Put $K:=h^{-1}(\mathscr{K})$ and $O:=h^{-1}(\mathcal{O})$. An arbitrary element $\gamma \in \Omega(K, O) \subseteq$ $\operatorname{Aut}^{1}(\mathscr{L})$ satisfies the relations

$$
\gamma(K) \subseteq O \subseteq h^{-1}(\mathscr{U})=: U
$$

and

$$
h \gamma h^{-1}(\mathscr{K}) \subseteq \mathscr{U}
$$

Hence, the set $\mathscr{K}$ is contained in the domain $\mathscr{W}$ of the map $h \gamma h^{-1}$. By Theorem (2.1) this implies

$$
\left.h \gamma h^{-1}\right|_{\mathscr{K}}=\left.\left(h \gamma h^{-1}\right)^{\prime}(0)\right|_{\mathscr{K}}
$$


and

$$
\Psi(\gamma)(\mathscr{K})=\left(h \gamma h^{-1}\right)^{\prime}(0)(\mathscr{K})=h \gamma h^{-1}(\mathscr{K}) \subseteq h\left(h^{-1}(\mathcal{O})\right)=\mathcal{O} .
$$

In particular,

$$
\Psi(\gamma) \in \Omega(\mathscr{K}, \mathcal{O})
$$

holds, and thus assertion (2) is proved.

(3) $\Psi: \operatorname{Aut}^{1}(\mathscr{L}) \rightarrow \Psi\left(\operatorname{Aut}^{1}(\mathscr{L})\right)$ is an open map.

Let $\tau_{c}$ denote the compact-open topology on $\operatorname{Aut}^{1}(\mathscr{L})$ and let $\tau_{\imath}$ denote the initial topology on $\operatorname{Aut}^{1}(\mathscr{L})$ with respect to the map $\Psi: \operatorname{Aut}^{1}(\mathscr{L}) \rightarrow \mathrm{GL}_{n} \mathbb{R}$. Set $\Gamma:=\left(\operatorname{Aut}^{1}(\mathscr{L}), \tau_{2}\right)$. Then $\Psi$ is a homeomorphism of $\Gamma$ onto $\Psi(\Gamma)$ by Corollary (2.2). In order to verify the openness of $\Psi$, we show that the topology $\tau_{\imath}$ is finer than the compact-open topology $\tau_{c}$. Since $L$ is locally compact, the topology $\tau_{c}$ is the coarsest topology on $L$ such that the evaluation mapping

$$
\operatorname{Aut}^{1}(\mathscr{L}) \times L \rightarrow L:(\gamma, x) \mapsto \gamma(x)
$$

is continuous, see, e.g., [5, p. 224]. Hence it suffices to verify the continuity of the mapping $\eta: \Gamma \times L \rightarrow L:(\gamma, x) \mapsto \gamma(x)$. The group $\Gamma$ has a countable base since it is a subgroup of $\mathrm{GL}_{n} \mathbb{R}$. So, it suffices to verify the sequential continuity of $\eta$.

For this, choose a compact neighborhood $\mathscr{C}$ of 0 such that $\mathscr{C} \subset \mathscr{U l}$, and select an open star-shaped neighborhood $\mathcal{O}$ of 0 satisfying $\bar{O} \subset \mathscr{C}^{\circ}$. Furthermore, let $\mathscr{K}$ be a compact star-shaped neighborhood of 0 with $\mathscr{K} \subset \mathcal{O}$. Set $C:=h^{-1}(\mathscr{C})$ and $K:=h^{-1}(\mathscr{K})$. Then $\Omega:=\Psi^{-1}(\Omega(\mathscr{K}, \mathcal{O}))$ is a neighborhood of 1 in $\Gamma$. Let $W_{\gamma}$ denote the domain of the map $\gamma \in \Omega$. The next step is to verify the inclusion

$$
K \subseteq W:=\bigcap_{\gamma \in \Omega} W_{\gamma}
$$

Suppose that this inclusion does not hold. Then there would exist an automorphism $\gamma \in \Omega$ and an element $x \in K \backslash W_{\gamma}$. This would imply that $\gamma(x) \notin U$ by Theorem (2.1). Set $y:=h(x)$ and consider the path $\rho:[0,1] \rightarrow \mathbb{R}^{n}: t \mapsto t \cdot y$ connecting the element 0 and $y$. Since $\mathscr{K}$ is star-shaped and $y=h(x)$ lies in $h(K)=\mathscr{K}$, this implies that $\rho([0,1])$ lies in $\mathscr{K}$. Hence $h^{-1} \rho$ is a path from 0 to $x=h^{-1}(y)$ which is contained in $U$. Moreover, the map $\gamma h^{-1} \rho$ is a path between 0 and $\gamma h^{-1}(y) \notin U$. Because $\partial C$ separates the point 0 from $L \backslash U$, there is thus an element $t_{c} \in[0,1]$ such that

$$
\gamma h^{-1}\left(t_{c} \cdot y\right) \in \partial C \subseteq U
$$


In particular, this implies $t_{c} \cdot y \in W_{\gamma}$ and $\Phi_{y}\left(t_{c} \cdot y\right)=h y h^{-1}\left(t_{c} \cdot y\right) \in \partial \mathscr{C}$. On the other hand, by Theorem (2.1) we have

$$
\Phi_{\gamma}\left(t_{c} \cdot y\right)=\Phi_{\gamma}^{\prime}(0)\left(t_{c} \cdot y\right)=\Psi(\gamma)\left(t_{c} \cdot y\right)=t_{c} \cdot \Psi(\gamma)(y) .
$$

Now $y \in \mathscr{K}$ and $\Psi(\gamma) \in \Omega$ imply that $\Psi(\gamma)(y)$ is contained in $\mathcal{O}$. Because $\mathcal{O}$ is star-shaped, the last computation shows that $\Phi_{\gamma}\left(t_{c} \cdot y\right)$ lies in $\mathcal{O}$. But this contradicts the fact that $\mathscr{O} \cap \partial \mathscr{C}=\varnothing$ holds. Hence, this proves the inclusion $K \subseteq W$.

In particular, the set $W$ is a neighborhood of the element 0 . Now we proceed to verify the continuity of the map $\eta$ on $\Omega \times W^{\circ}$. For this choose an element $x \in W^{\circ}$ and let $\left(x_{n}\right)_{n \in \mathbb{N}}$ be a sequence in $L$ satisfying $\lim _{n \rightarrow \infty} x_{n}=x$. Furthermore, select an automorphism $\gamma \in \Omega$ and let $\left(\gamma_{n}\right)_{n \in \mathbb{N}}$ be a sequence in $\Gamma$ with $\lim _{n \rightarrow \infty} \gamma_{n}=\gamma$. Since $W^{\circ}$ and $\Omega$ are open sets, we may choose the sequence $\left(x_{n}\right)_{n \in \mathbb{N}}$ in $W$ and the sequence $\left(\gamma_{n}\right)_{n \in \mathbb{N}}$ in $\Omega$. Noting that the evaluation map of $\mathrm{GL}_{n} \mathbb{R}$ is continuous, this implies

$$
\begin{aligned}
h\left(\lim _{n \rightarrow \infty} \eta\left(\gamma_{n}, x_{n}\right)\right) & =h\left(\lim _{n \rightarrow \infty} \gamma_{n}\left(x_{n}\right)\right)=\lim _{n \rightarrow \infty} h \gamma_{n}\left(x_{n}\right)=\lim _{n \rightarrow \infty} h \gamma_{n} h^{-1} h\left(x_{n}\right) \\
& =\lim _{n \rightarrow \infty} \Phi_{\gamma_{n}}\left(h\left(x_{n}\right)\right)=\lim _{n \rightarrow \infty} \Psi\left(\gamma_{n}\right)\left(h\left(x_{n}\right)\right)=\Psi(\gamma)(h(x)) \\
& =\Phi_{\gamma}(h(x))=h(\gamma(x)),
\end{aligned}
$$

and thus $\lim _{n \rightarrow \infty} \eta\left(\gamma_{n}, x_{n}\right)=\gamma(x)$ holds. Hence the evaluation map $\eta$ is continuous at $(\gamma, x)$.

Now select an element $\gamma \in \Omega$ and a sequence $\left(\gamma_{n}\right)_{n \in \mathbb{N}}$ in $\Omega$ with $\lim _{n \rightarrow \infty} \gamma_{n}=\gamma$, where the limit is taken with respect to the topology $\tau_{l}$. The set $R:=$ $\left\{x \in L ; \lim _{n \rightarrow \infty} \gamma_{n}(x)=\gamma(x)\right\}$ is a subloop of $L$ which contains the open set $W^{\circ}$. By [3, (3.1), (3.2)], this implies that $R$ is an open as well as a closed subset of $L$. Thus we have $R=L$, since $L$ is connected. We shall use this identity to verify the continuity of $\eta$ on the whole set $\Gamma \times L$. For this fix a pair $(\gamma, x) \in \Gamma \times L$ and select sequences $\left(\gamma_{n}\right)_{n \in \mathbb{N}}$ in $\Gamma$ and $\left(x_{n}\right)_{n \in \mathbb{N}}$ in $L$ satisfying

$$
\lim _{n \rightarrow \infty} \gamma_{n}=\gamma, \quad \lim _{n \rightarrow \infty} x_{n}=x .
$$

The equations $\gamma_{n}=\gamma \lambda_{n}$ and $x_{n}=x+y_{n}$ uniquely determine sequences $\left(\lambda_{n}\right)_{n \in \mathbb{N}}$ in $\Gamma$ and $\left(y_{n}\right)_{n \in \mathbb{N}}$ in $L$ satisfying

$$
\lim _{n \rightarrow \infty} \lambda_{n}=1, \quad \lim _{n \rightarrow \infty} y_{n}=0,
$$

where we may assume without loss of generality that $y_{n} \in W^{\circ}$ holds for all 
$n \in \mathbb{N}$. Using the continuity of $\eta$ at the points of $\{0\} \times W^{\circ}$ and remembering the identity $R=L$, we obtain

$$
\begin{aligned}
\lim _{n \rightarrow \infty} \eta\left(\gamma_{n}, x_{n}\right) & =\lim _{n \rightarrow \infty} \gamma_{n}\left(x_{n}\right)=\lim _{n \rightarrow \infty} \gamma \lambda_{n}\left(x+y_{n}\right)=\gamma\left(\lim _{n \rightarrow \infty}\left(\lambda_{n}(x)+\lambda_{n}\left(y_{n}\right)\right)\right) \\
& =\gamma\left(\lim _{n \rightarrow \infty} \lambda_{n}(x)\right)+\gamma\left(\lim _{n \rightarrow \infty} \lambda_{n}\left(y_{n}\right)\right)=\gamma(x)+\gamma(0)=\gamma(x) .
\end{aligned}
$$

This proves that the evaluation map $\eta$ is continuous at $(\gamma, x)$. Thus $\Psi$ is a homeomorphism between $\operatorname{Aut}^{1}(\mathscr{L})$ and $\Psi\left(\operatorname{Aut}^{1}(\mathscr{L})\right)$, and the proof is complete.

Knowing that the group $\operatorname{Aut}^{1}(\mathscr{L})$ is embedded as a topological group into $\mathrm{GL}_{n} \mathbb{R}$, the question arises of how the action of $\operatorname{Aut}^{1}(\mathscr{L})$ on $\mathscr{L}$ is related with the action of $\Psi\left(\operatorname{Aut}^{1}(\mathscr{L})\right)$ on $\mathbb{R}^{n}$.

(2.4) LEMMA. Let $\mathscr{L}=(L, 0,+)$ be a smooth loop of dimension $n$. Let $h: U \rightarrow \mathbb{R}^{n}$ be a canonical coordinate system of $\mathscr{L}$ according to Theorem (1.5) with induced loop $\left(\mathbb{R}^{n}, \mathscr{U}, 0, \oplus\right)$. Let $\mathscr{U}^{*} \subseteq \mathscr{U}$ be a star-shaped neighborhood of 0 . Let $\Psi: \operatorname{Aut}^{1}(\mathscr{L}) \rightarrow \mathrm{GL}_{n} \mathbb{R}$ be the embedding defined in Corollary (2.3). Let $\left(\gamma_{n}\right)_{n \in \mathbb{N}}$ be a sequence in $\operatorname{Aut}^{1}(\mathscr{L})$ and let $u \in h^{-1}\left(\mathscr{U}^{*}\right) \subseteq U$. Then the following statements are equivalent

(i) $\lim _{n \rightarrow \infty} \gamma_{n}(u)=0$.

(ii) $\lim _{n \rightarrow \infty} \Psi\left(\gamma_{n}\right)(h(u))=0$.

Proof. Let $\mathscr{W}_{n} \subset \mathbb{R}^{n}$ denote the domain of the map $\Phi_{\gamma_{n}}$ and set $W_{n}:=h^{-1}\left(\mathscr{W}_{n}\right)$ for all $n \in \mathbb{N}$. First, let $\lim _{n \rightarrow \infty} \gamma_{n}(u)=0$. We may assume that $\gamma_{n}(u) \in U$ for all $n \in \mathbb{N}$. By Theorem (2.1) this implies that $u \in W_{n}$ and thus $h(u) \in \mathscr{W}_{n}$ holds for all $n \in \mathbb{N}$. Consequently, we obtain

$\lim _{n \rightarrow \infty} \Psi\left(\gamma_{n}\right)(h(u))=\lim _{n \rightarrow \infty} \Phi_{\gamma_{n}}(h(u))=\lim _{n \rightarrow \infty} h \gamma_{n}(u)=h\left(\lim _{n \rightarrow \infty} \gamma_{n}(u)\right)=h(0)=0$.

Conversely, assume that $\lim _{n \rightarrow \infty} \Psi\left(\gamma_{n}\right)(h(u))=0$. Set $v:=h(u)$. Choose a compact neighborhood $\mathscr{C} \subset \mathscr{U}^{*}$ of 0 and set $C:=h^{-1}(\mathscr{C})$. The map $\rho:[0,1] \rightarrow \mathbb{R}^{n}: t \mapsto t \cdot v$ is a path from 0 to $v$. Since $\mathscr{U}^{*}$ is star-shaped, $\rho([0,1]) \subset \mathscr{U}^{*}$ and thus $h^{-1} \rho$ is a path starting in 0 and ending in $h^{-1}(v)$, which is contained in $h^{-1}\left(\mathscr{U}^{*}\right) \subseteq U$.

The element $u$ is contained in almost all $W_{n}$, for otherwise there is a sequence $\left(n_{k}\right)$ of integers such that $u \notin W_{n_{k}}$. By Theorem (2.1) this implies that $\gamma_{n_{k}}(u) \notin U$ for all $k \in \mathbb{N}$. Now $\gamma_{n_{k}} h^{-1} \rho$ is a path between 0 and $\gamma_{n_{k}}(u)$. Since the element $\gamma_{n_{k}}(u)$ is not contained in $U$, and because the set $\partial C$ separates the 
element 0 and the set $L \backslash U$, there exists some element $t_{k} \in[0,1]$ such that $\gamma_{n_{k}} h^{-1}\left(t_{k} \cdot v\right) \in \partial C$. Because of $\partial \mathscr{C} \subset \mathscr{U}$, this implies that $t_{k} \cdot v \in \mathscr{W}_{n_{k}}$, and hence we obtain

$$
\Psi\left(\gamma_{n_{k}}\right)\left(t_{k} \cdot v\right)=\Phi_{\gamma_{n_{k}}}\left(t_{k} \cdot v\right)=h\left(\gamma_{n_{k}} h^{-1}\left(t_{k} \cdot v\right)\right) \in h(\partial C)=\partial \mathscr{C}
$$

On the other hand, we have

$$
\begin{aligned}
\lim _{k \rightarrow \infty} \Psi\left(\gamma_{n_{k}}\right)\left(t_{k} \cdot h(u)\right) & =\lim _{k \rightarrow \infty} t_{k} \cdot \Psi\left(\gamma_{n_{k}}\right)(h(u)) \\
& =\lim _{k \rightarrow \infty} t_{k} \cdot \lim _{k \rightarrow \infty} \Psi\left(\gamma_{n_{k}}\right)(h(u)) \\
& =\lim _{k \rightarrow \infty} t_{k} \cdot 0 \\
& =0 .
\end{aligned}
$$

This contradicts $0 \notin \overline{\partial \mathscr{C}}$. Hence $u$ is contained in almost all neighborhoods $W_{n}$ and by Theorem (2.1) we finally conclude that

$$
h\left(\lim _{n \rightarrow \infty} \gamma_{n}(u)\right)=\lim _{n \rightarrow \infty} h \gamma_{n}(u)=\lim _{n \rightarrow \infty} \Phi_{\gamma_{n}}(h(u))=\lim _{n \rightarrow \infty} \Psi\left(\gamma_{n}\right)(h(u))=0
$$

(2.5) DEFINITION. Let $\mathscr{D}=(D, 0,1,+, \circ)$ be a topological double loop. If the additive loop of $\mathscr{D}$ possesses a (smooth) localization, then $\mathscr{D}$ is called a smooth double loop.

(2.6) DEFINITION. Let $\mathscr{D}=(D, 0,1,+, \circ)$ be a smooth double loop. The group of all continuous automorphisms of $\mathscr{D}$ lying in $\operatorname{Aut}^{1}(D, 0,+)$ is denoted by $\operatorname{Aut}^{1}(\mathscr{D})$.

Note that the last two definitions stress the peculiar role of the neutral element 0 of a double loop $\mathscr{D}$. The following proofs are based on the fact that the automorphism group Aut ${ }^{1}(\mathscr{D})$ can be embedded in a linear group, which acts on the tangential space of the element 0 .

(2.7) LEMMA. If $\mathscr{L}=(L, 0,+)$ is a connected smooth loop, then $\mathrm{Aut}^{1}(\mathscr{L})$ is a closed subgroup of $\operatorname{Aut}(\mathscr{L})$.

Proof. Let $\left(\gamma_{n}\right)_{n \in \mathbb{N}}$ be a sequence in $\operatorname{Aut}^{1}(\mathscr{L})$ converging to an element $\gamma$ in $\operatorname{Aut}(\mathscr{L})$. Since the group $\operatorname{Aut}(\mathscr{L})$ is taken with the compact-open topology, the sequence $\left(\gamma_{n}\right)_{n \in \mathbb{N}}$ converges pointwise to $\gamma$. Let $\mathscr{L}_{U}$ be a smooth localization of $\mathscr{L}$ with associated canonical coordinate system $h$ and induced loop $\left(\mathbb{R}^{n}, \mathscr{U}, 0, \oplus\right)$. Because $\gamma$ is a continuous map, there is a compact neighborhood $K$ of 0 contained in $U$ such that $\gamma(K) \subseteq U$ holds. Because of $\lim _{n \rightarrow \infty} \gamma_{n}=$ $\gamma$ and the compactness of $K$, we may assume that $\gamma_{n}(K) \subseteq U$ holds for every 
$n \in \mathbb{N}$. In particular, the mappings $\Phi_{\gamma_{n}}$ are defined on the set $K$. Thus by Theorem (2.1), this implies that

$$
\left.\Phi_{\gamma_{n}}\right|_{K}=\left.\Phi_{\gamma_{n}}{ }^{\prime}(0)\right|_{K}
$$

holds for every $n \in \mathbb{N}$. Applying Corollary (2.3), we conclude that the sequence of the restricted functions $\left(\left.\Phi_{\gamma_{n}}\right|_{h(K)}\right)_{n \in \mathbb{N}}$ converges pointwise. Now, the set $h(K)$ is a neighborhood of 0 in $\mathbb{R}^{n}$ and the mappings $\Phi_{\gamma_{n}}^{\prime}(0)$ are linear. Hence, the sequence $\left(\Phi_{\gamma_{n}}{ }^{\prime}(0)\right)_{n \in \mathbb{N}}$ converges in $G_{n} \mathbb{R}$ to a linear mapping $\lambda$. By definition of $\gamma$ we infer that

$$
\Phi_{\gamma} \operatorname{lh}_{(K)}=\left.\lambda\right|_{h(K)},
$$

i.e. the map $\Phi_{\gamma}$ is analytical on $h(K)$. Thus the automorphism $\gamma$ is continuously differentiable on an appropriate neighborhood of 0 , since $h$ and $h^{-1}$ are smooth mappings. Finally, this implies $\gamma \in \operatorname{Aut}^{1}(\mathscr{L})$, which proves the lemma.

As an immediate consequence of the last lemma we get the following result.

(2.8) PROPOSITION. If $\mathscr{D}=(D, 0,1,+, \circ)$ is a connected smooth double loop, then the group Aut $^{1}(\mathscr{D})$ is locally compact.

Proof. Let $\mathscr{L}=(D, 0,+)$ be the additive loop of $\mathscr{D}$. Because of the representation $\operatorname{Aut}(\mathscr{D})=\{\gamma \in \operatorname{Aut}(\mathscr{L}) ; \gamma(x \circ y)=\gamma(x) \circ \gamma(y)$ for all $x, y \in D\}$, the group $\operatorname{Aut}(\mathscr{D})$ is a closed subgroup of $\operatorname{Aut}(\mathscr{L})$. By Lemma (2.7) this implies that the group $\operatorname{Aut}^{1}(\mathscr{D})$, which can be written as the intersection $\operatorname{Aut}^{1}(\mathscr{D})=\operatorname{Aut}(\mathscr{D}) \cap \operatorname{Aut}^{1}(\mathscr{L})$, is closed in $\operatorname{Aut}(\mathscr{D})$. Since this last group is locally compact by [1], the claim of the proposition follows.

(2.9) LEMMA. Let $\mathscr{D}=(D, 0,1,+, \circ)$ be a connected smooth double loop and let $\left(\gamma_{n}\right)_{n \in \mathbb{N}}$ be a sequence in Aut ${ }^{1}(\mathscr{D})$. Then there is a subsequence $\left(\gamma_{n}^{\prime}\right)_{n \in \mathbb{N}}$ of $\left(\gamma_{n}\right)_{n \in \mathbb{N}}$ such that the set $N:=\left\{x \in D ; \lim _{n \rightarrow \infty} x^{\gamma_{n}^{\prime}}=0\right\}$ is not dense in $D$.

Proof. Let $\Psi: \Gamma \rightarrow \mathrm{GL}_{n} \mathbb{R}$ be the embedding of Corollary (2.3) associated to a fixed canonical coordinate system $h: U \rightarrow \mathbb{R}^{n}$ with induced loop $\left(\mathbb{R}^{n}, \mathscr{U}, 0, \oplus\right)$. Set $\hat{D}:=D \cup\{\infty\}$.

Assume that $N$ is dense in $D$. Let $\mathscr{U}^{*} \subseteq \mathscr{U}$ be a star-shaped open neighborhood of 0 and set $U^{*}:=h^{-1}\left(\mathscr{U}^{*}\right)$. Then $\overline{U^{*} \cap N}=U^{*}$ and there are linearly independent vectors $e_{1}, \ldots, e_{n}$ in $h\left(U^{*} \cap N\right) \subseteq \mathscr{U}^{*}$. Setting $f_{i}:=h^{-1}\left(e_{i}\right) \in U^{*}$ we obtain

$$
\lim _{n \rightarrow \infty} \gamma_{n}^{\prime}\left(f_{i}\right)=0 .
$$

Applying Lemma (2.4) we obtain

$$
\lim _{n \rightarrow \infty} \Psi\left(\gamma_{n}^{\prime}\right)\left(e_{i}\right)=0 .
$$


Since the mappings $\Psi\left(\gamma_{n}^{\prime}\right)$ are linear and the elements $e_{1}, \ldots, e_{n}$ form a basis of $\mathbb{R}^{n}$, this implies that

$$
\lim _{n \rightarrow \infty} \Psi\left(\gamma_{n}^{\prime}\right)(x)=0
$$

holds for any $x \in \mathscr{U}^{*}$. Applying Lemma (2.4) once again, we infer that

$$
\lim _{n \rightarrow \infty} \gamma_{n}^{\prime}(u)=0
$$

for any $u \in U^{*}$. Hence $U^{*} \subseteq N$ and consequently $U^{*-1} \subseteq N^{-1}$. The set $U^{*-1} \backslash\{\infty\}$ is open in $D$ (see $[2, \mathrm{XI} .8 .3]$ ) and disjoint to $N$, since $N \cap N^{-1}=\varnothing$. But then $N$ cannot be dense in $D$, a contradiction.

The following is the central result of this paper.

(2.10) THEOREM. If $\mathscr{D}=(D, 0,1,+, \circ)$ is a connected smooth double loop, then the group $\operatorname{Aut}^{1}(\mathscr{D})$ is compact.

Proof. Let $\mathscr{D}_{U}=(D, U, 0,+)$ be a smooth localization of $\mathscr{D}$. Set $\Gamma:=\operatorname{Aut}^{1}(\mathscr{D})$ and let $\Psi: \operatorname{Aut}^{1}(\mathscr{D}) \rightarrow \mathrm{GL}_{n} \mathbb{R}$ denote the embedding defined in Corollary (2.3) which corresponds to a fixed canonical coordinate system $h$ of $\mathscr{D}_{v}$. Let $\left(\mathbb{R}^{n}, \mathscr{U}, 0, \oplus\right)$ be the induced loop of $h$.

First, we show that all orbits of $\Gamma$ in $D$ are bounded, i.e. they are relatively compact sets. Suppose that there is an unbounded orbit $u^{\Gamma}$ in $D$. Then there exists a sequence $\left(\gamma_{n}\right)_{n \in \mathbb{N}}$ in $\Gamma$ with $\lim _{n \rightarrow \infty} u^{\gamma_{n}}=\infty$. Setting $v:=u^{-1}$ this implies that $\lim _{n \rightarrow \infty} v^{\gamma_{n}}=0$. Similarly to the proof in [1] we may choose a subsequence $\left(\gamma_{n}^{*}\right)_{n \in \mathbb{N}}$ of $\left(\gamma_{n}\right)_{n \in \mathbb{N}}$ and a dense subset $R$ in $D$ such that $\lim _{n \rightarrow \infty} x^{\gamma_{n}^{*}}$ exists in $D$ for any $x \in R$. We denote this subsequence again by $\left(\gamma_{n}\right)_{n \in \mathbb{N}}$. Moreover, the set $N:=\left\{x \in D ; \lim _{n \rightarrow \infty} x^{y_{n}}=0\right\}$ is not dense in $D$ by Lemma (2.9). The mapping $x \mapsto v \circ x: D \rightarrow D$ is a homeomorphism by the very definition of a double loop, since $v \neq 0$. Hence, also the set $v \circ R$ is dense in $D$. But this implies that the set $N$ is dense in $D$, because of the relation $v \circ R \subseteq N$. This, however, contradicts the result of Lemma (2.9). Consequently, all orbits of $\Gamma$ are bounded in $D$.

By Lemma (2.4) and what was proved above there is a star-shaped neighborhood $\mathscr{U}^{*} \subseteq \mathscr{U}$ such that the orbits $w^{\Psi_{(\Gamma)}}$ are bounded for every $w \in \mathscr{U}^{*}$. Let $e_{1}, \ldots, e_{n} \in \mathscr{U}^{*}$ be linearly independent elements. In particular, the orbits of $e_{i}$ are bounded. Thus there is a compact neighborhood $\mathscr{K} \subseteq \mathbb{R}^{n}$ of 0 satisfying $e_{i}^{\Psi(\Gamma)} \subseteq \mathscr{K}$ for any $i \in\{1, \ldots, n\}$. The elements of $\Psi(\Gamma)$ are linear mappings. Consequently, there is a neighborhood $\mathcal{O} \subseteq \mathbb{R}^{n}$ of 0 with $\mathcal{O}^{\Psi(\Gamma)} \subseteq \mathscr{K}$. If $\mathscr{W}^{\prime}$ is an arbitrary neighborhood of 0 in $\mathbb{R}^{n}$, then by the compactness of $\mathscr{K}$ there is a positive real number $\delta$ such that $\delta \mathscr{K} \subseteq \mathscr{W}^{\prime}$ holds. 
This implies that

$$
(\delta \mathcal{O})^{\Psi(\Gamma)}=\delta\left(\mathcal{O}^{\Psi(\Gamma)}\right) \subseteq \delta \mathscr{K} \subseteq \mathscr{W}^{\prime}
$$

Being locally compact, the group $\Psi(\Gamma)$ is closed in $\mathrm{GL}_{n} \mathbb{R}$, and the compactness of $\Psi(\Gamma)$ follows by the Arzela-Ascoli compactness criterion. Finally, the compactness of $\Gamma$ follows from Corollary (2.3).

\section{REFERENCES}

1. Bödi, R., 'Automorphism groups of locally compact connected double loops are locally compact' (to appear in Arch. Math.).

2. Grundhöfer, T. and Salzmann, H., 'Locally compact double loops and ternary fields,' in Quasigroups and Loops: Theory and Applications (eds O. Chein et al.), Heldermann, Berlin, 1990.

3. Hofmann, K. H., 'Topologische Loops', Math. Z. 70 (1958), 13-37.

4. Holmes, J. P. and Sagle, A. A., 'Analytic $H$-spaces, Campbell-Hausdorff formula, and alternative algebras', Pacific J. Math. 91 (1980), 105-134.

5. Kelley, J. L., General Topology, Van Nostrand, Princeton, N.J., 1955.

6. Kozma, J., 'Behaviour of loops in a canonical coordinate system', Arch. Math. 55 (1990), 498502 .

7. Kozma, J., 'On the differentiability of loopmultiplication in canonical coordinate-system', Publ. Math. Debrecen. 37 (1990), 313-325.

8. Salzmann, H., 'Kompakte 8-dimensionale projektive Ebenen mit großer Kollineationsgruppe', Math. Z. 176 (1981), 345-357.

Author's address:

Richard Bödi,

Mathematisches Institut, Universität Tübingen,

Auf der Morgenstelle 10, D-7400 Tübingen,

Germany.

(Received, August 22, 1992) 